\title{
Micro Satellite Thermal Balance Testing: Orbit Heat Flux Simulation Method and Verification
}

\author{
Liu Jia ${ }^{1}$,Li Ming ${ }^{1}$ and Gao Qinghua ${ }^{2}$ \\ ${ }^{1}$ DFH satellite Co., LTD, Beijing 100094, China \\ ${ }^{2}$ Beijing Institute of Spacecraft Environment Engineering, Beijing 100094, China;
}

\begin{abstract}
Comparing to conventional satellites, micro satellite features smaller envelop size, lower cost, and shorter R\&D period. At the same time, while micro satellite generally runs within the LEO, it is more susceptible to the earth IR radiation. To simulate the orbital heat flux efficiently for the micro satellite, in this paper the thermal balance testing respectively using infrared cage and solar simulator are performed. The resulting differences between the two simulating approaches are examined with care. Conclusions can be drawn from analysis: in thermal balance testing of micro satellite, in terms of simulating orbital heat flux, the results of two simulators are close enough to consider that it is feasible to choose infrared cage as orbital heat flux simulator, given thermal coating material of typical IR emittance and solar absorptance ratio. Results demonstrate that by using infrared cage simulator, the actual temperature is about $5^{\circ} \mathrm{C}$ higher than theoretical value. The background heat flux interference stays stable at $17 \%$ of intensity of simulated orbital heat flux, and the value could be referred to in the thermal mathematical model modification.
\end{abstract}

\section{Introduction}

Driven by rapid growth of technical innovation and demand for astronautic task, researches and developments of satellites are required to be both lowcost and short-period, which are met by the emergence of micro satellite. However, design of thermal control subsystem in spacecraft is challenged by complexity and uncertainty introduced by mission complexity. In order to verify both scheme and assembly of thermal control subsystem, it is crucial to perform the ground thermal balance testing and obtain on-board temperature data $[1$, 2, and 3].

In contrast to conventional satellites, micro satellite features smaller envelop size, lower cost, and shorter $\mathrm{R} \& \mathrm{D}$ period. At the same time, while micro satellite generally runs within the LEO, it is more susceptible to the earth IR radiation. The contribution of this paper are: a) studies on orbit heat flux simulation method for ground thermal balance testing of micro satellite; b) performing two different types of ground thermal balance testing based on infrared cage and solar simulator respectively; c) analysing the difference between the results of two simulations.

\section{Options for ground simulation of orbit heat flux}

Methods for ground simulation of orbit heat flux can be sorted into two categories: incident and absorptive.
Specifically, incident simulator (such as solar simulator) mimics parameters of orbit heat flux including irradiance, directions and spectral characteristics. Meanwhile, absorptive simulator uses heating devices (such as infrared cage, heater, etc.) to imitate orbital heat absorbed by the surface of satellite. In contrast to conventional satellites, micro satellite features smaller envelop size, lower cost, and shorter R\&D period. At the same time, while micro satellite generally runs within the LEO, it is more susceptible to the earth IR radiation. To simulate orbit heat flux efficiently, the following approaches are studied $[4,5]$ :

Solar Simulator. For small-sized spacecraft or units, solar simulator is the most appropriate option. Nevertheless, in micro satellite's case, the system is susceptible to earth IR radiation, the solar simulator fails to simulate earth IR, whereas it can only provide solar spectrum. In addition, the lower budget of micro satellite prohibits solar simulator due to the high running expenditure.

Foil Heater. This method simulates orbit heat flux by sticking foil heaters onto exterior surface panel. The advantage is to enable transient simulation, and to facilitate the modification of thermal mathematic model. But concerning micro satellite's short R\&D period, the thermal balance testing is only performed during the flight-ready phrase (rather than the missing CDR phase), thus incurs two problems below: 1) limited by practical situation, the mocked surface panels are usually used for the testing, therefore radiative and conductive 
characteristics of the real panels can only be theoretically assumed, instead of being verified by the testing; 2) after the thermal balance testing, the mocked panels need to be replaced by the real ones, which raises the $R \& D$ cost as well as reliability risk.

Infrared Cage. The cage is an enveloped frame with several belts twisted on it. The energy is generated by heated stainless steel band, and the absorptive flux density is measured with radiometer on the satellite surface. The cage is split to several areas as heat fluent. The belt on an area is one chain and will be electrified during test. Whenever it powered on, heat will emit as infrared energy. Different area will be put different current as heat fluent in orbit. The power can be modified in each case we want to simulate during test.

Cold Plate. Unlike the infrared cage, surface of cold plate can be refrigerated or be heated, depending on the demand. By surrounding the satellite with cold plate, this simulation method is able to eliminate interference caused by background heat flux, which helps improving the thermal mathematic model. Additionally, the approach allows using all flight-ready parts and devices, hence leads to the most accurate simulation results. The shortcomings with cold plate simulation are requirements for larger vacuum simulator and plenty of assembly instruments. Moreover, to achieve ideal thermal uniformity, the simulator demand for larger sized cold plates and satellite, compare to the IR cage method.

To sum up, as to micro satellite, the infrared cage simulator turns out to be the most feasible and economical option. In this paper, infrared cage simulation is used to modify the thermal mathematical model, and the model is taken to predict the on orbit status. To verify the validity of infrared cage simulator, the difference between the results of both IR cage and solar simulator is examined.

\section{Testing procedure}

\subsection{Testing Objects}

The objects are two pieces of $200 \mathrm{~mm} \times 200 \mathrm{~mm} \times 1 \mathrm{~mm}$ aluminum alloy plates, each weighs approximately 111.2 gram. The faced sides are painted with SR107-ZK white thermal coating $(\mathrm{a}=0.17, \varepsilon=0.87)$ and aluminum Kapton film $(a=0.36, \varepsilon=0.69)$ respectively. The back sides are both covered with $25 \mu \mathrm{m}$ aluminum Mylar, and optical parameters are taken as beginning of life. The objects are as illustrated in Fig. 1.

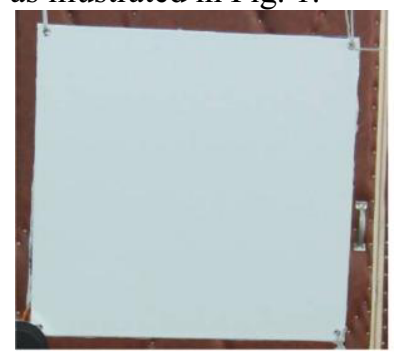

SR107-ZK White Painting

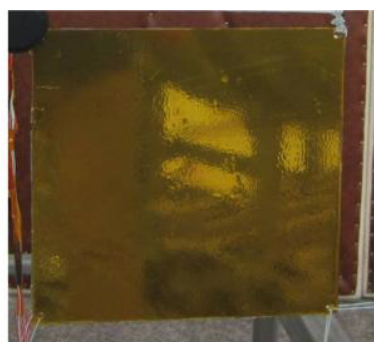

Aluminized Kapton Blanket
Figure 1. Tested Objects

\subsection{Testing process}

To analyze different effects from infrared cage and solar simulator, the thermal balance testing is performed in two phases.

In the solar simulator testing phase, the optical system of solar simulator and other parts would generate infrared radiation to the testing objects, thus necessitates radiometer to measure actual flux density that arrives at the objects.

Heat flux that absorbed by radiometer is written as:

$$
q_{0}=\alpha_{s} S+\varepsilon_{H} q_{1}=\varepsilon_{H} \sigma T^{4}
$$

Heat flux that arrives at radiometer, i.e. arrives at the object, is written as:

$$
q_{H}=\frac{q_{0}}{\varepsilon_{H}}=\frac{\alpha_{s}}{\varepsilon_{H}} S+q_{1}=\sigma T^{4}
$$

where $q_{0}$ denotes the absorbed heat flux of radiometer $\left(\mathrm{W} / \mathrm{m}^{2}\right) ; q_{H}$ is heat flux that arrives at radiometer; $q_{I}$ is infrared radiation caused by the system $\left(\mathrm{W} / \mathrm{m}^{2}\right) ; S$ is solar irradiation $\left(1 \mathrm{~S}_{0}\right.$ is $\left.1410 \mathrm{~W} / \mathrm{m}^{2}\right) ; a_{S}$ is the absorptance of radiometer black surface, as $0.93 ; \varepsilon_{H}$ the emittance of radiometer black surface, as $0.88 ; T$ is the temperature of radiometer $(\mathrm{K})$.

In contrast, in the infrared cage phase, the arrived heat flux to be simulated consists of not only radiation from solar simulator, but also from the combination of the optical system of solar simulator and others, which is denoted by $q$ in above Eq. 2. In this phase, it is necessary to adjust the currency through the heating band, to match the target temperature on radiometer with the temperature $\mathrm{T}$ in solar simulator phase.

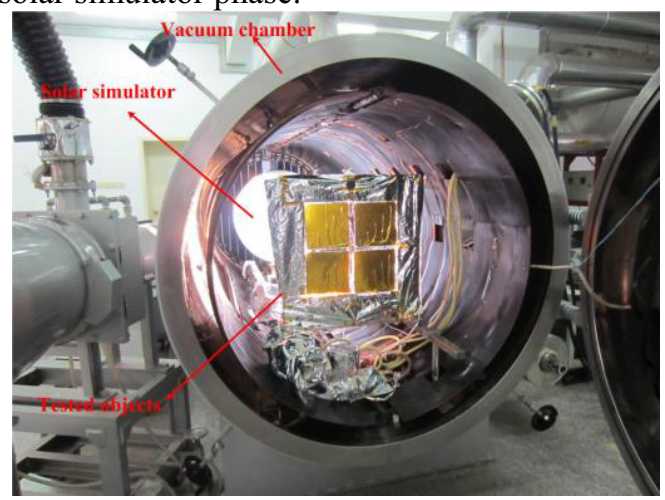

Figure2. Technical Status of solar simulator

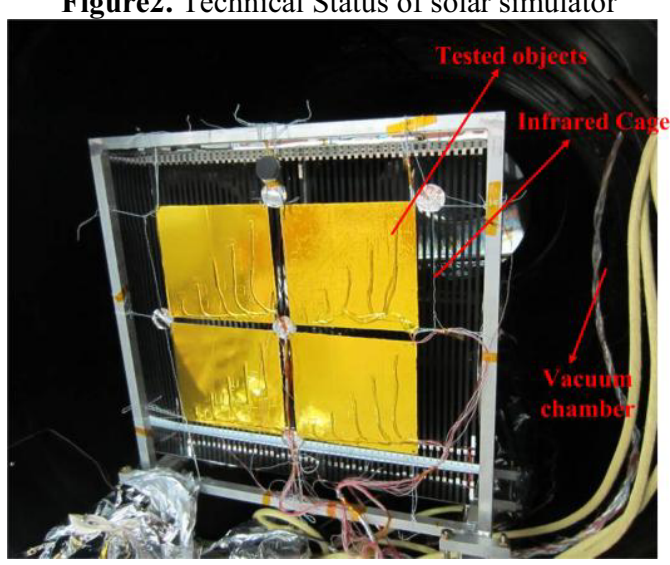

Figure3. Technical Status of infrared cage 
Testing status of both phases is demonstrated in Fig.2 and Fig.3, and the technical statuses are set to be as consistent as possible.

\subsection{Test results and analysis}

\subsubsection{The first phase thermal balance test}

The first phase thermal balance test was carried out in the vacuum chamber with solar simulator, assuming there are 3 sorts of external heating to effect on the tested objects:

a) Solar irradiation(S): $1 \mathrm{~S}_{0}$ is $1410 \mathrm{~W} / \mathrm{m}^{2}$, so simulated orbital heat flux are $0.5 \mathrm{~S}_{0}, 0.8 \mathrm{~S}_{0}, 1.0 \mathrm{~S}_{0}$;

b) The infrared radiation of faced side $\left(q_{1}\right)$ : Besides the solar irradiation, the infrared radiation from the chamber or optical system of solar simulator is considered which measured by 4 radiometers outside the facula.

c) The infrared radiation of revised side $\left(q_{2}\right)$ : To measure the background infrared radiation to test objects, 1 radiometer was installed on the revised side of test objects.

The energy balance for computing the temperature of surface is given by:

$$
C_{s} \frac{d T_{s}}{d t}=\alpha_{s} S+\varepsilon_{1} q_{1}+\varepsilon_{2} q_{2}-\left(\varepsilon_{1}+\varepsilon_{2}\right) \sigma T_{s}^{4}
$$

Where $C_{s}$ is the equivalent heat capacity of the tested object; $T_{s}$ is the temperature of the tested object; $\varepsilon_{1}, \varepsilon_{2}$ are the emittance of SR107-ZK white painting and aluminum Kapton film respectively; $\sigma$ is Stefan-Boltzmann constant.

According to the sampling of the first phase thermal balance test and calculation by Eq.3, the results are shown as Table 1 and Table 2. Note that Tested objects temperature $\left(T_{s}\right.$ ') means the average values of 4 sampling thermocouples; radiometer temperature $\left(T_{l}\right)$ in the faced side represents the average values of 5 sampling radiometers; radiometer temperature $\left(T_{2}\right)$ in the reversed side represents the average values of one sampling radiometer; and totally absorbed heat flux of the faced side denotes $\mathrm{q}_{0}$ You are free to use colour illustrations for the online version of the proceedings but any print version will be printed in black and white unless special arrangements have been made with the conference organiser. Please check with the conference organiser whether or not this is the case. If the print version will be black and white only, you should check your figure captions carefully and remove any reference to colour in the illustration and text. In addition, some colour figures will degrade or suffer loss of information when converted to black and white, and this should be taken into account when preparing them.

\subsubsection{The second phase thermal balance test}

The second phase thermal balance test was carried out in the vacuum chamber with infrared cage; there are just 2 sorts of external heating to effect on the tested objects.

The energy balance for computing the temperature of surface is given by:

$$
C_{s} \frac{d T_{s}}{d t}=\varepsilon_{1} q_{1}+\varepsilon_{2} q_{2}-\left(\varepsilon_{1}+\varepsilon_{2}\right) \sigma T_{s}^{4}
$$

According to the sampling and calculation of the first phase thermal balance test, the results are shown as Table 3 and Table 4.

\subsubsection{Analysis on results of two phases.}

Compare actual temperatures obtained from infrared cage phase to the ones from solar simulator, together with approximated calculated temperatures, conclusions can be drawn:

1. Speaking of micro satellite thermal balance testing with stable heat flux, in thermal balance testing of micro satellite, in terms of simulating orbital heat flux, the results of two simulators are close enough to consider that it is feasible to choose infrared cage as orbital heat flux simulator, given coating material of typical $\mathrm{a} / \varepsilon$ ratio. Results demonstrate that by using infrared cage simulator, the actual temperature is about $5^{\circ} \mathrm{C}$ higher than theoretical value.

2. Background heat flux interference generated by infrared cage is stronger than its counterpart from solar simulator. However, as to the same testing object, the background interference decreases while simulated heat flux goes up, which implies solar simulator is sensitive to optical characteristics of object's surface.

3. Comparing to background interference intensity of solar simulator increases from $5 \%$ to $15 \%$ of simulated heat flux while the latter falls from $1.0 \mathrm{~S}_{0}$ to $0.5 \mathrm{~S}_{0}$, the interference stays around $17 \%$ while infrared cage through the same process. Thus would facilitate the model modification.

\begin{tabular}{|c|c|c|c|c|c|c|c|c|c|}
\hline & \multicolumn{4}{|c|}{ Faced side } & \multicolumn{2}{|c|}{ Reverse side } & \multirow{2}{*}{$\begin{array}{c}\mathrm{T}_{\mathrm{s}}{ }^{\prime} \\
{\left[{ }^{\circ} \mathrm{C}\right]}\end{array}$} & \multirow{2}{*}{$\begin{array}{c}\mathrm{T}_{\mathrm{s}} \\
{\left[{ }^{\circ} \mathrm{C}\right]}\end{array}$} & \multirow[b]{2}{*}{$\Delta \mathrm{T}$} \\
\hline & $\mathrm{S}$ & $\begin{array}{c}\mathrm{T}_{1} \\
{\left[{ }^{\circ} \mathrm{C}\right]}\end{array}$ & $\begin{array}{c}\mathrm{q}_{1} \\
{\left[\mathrm{~W} / \mathrm{m}^{\wedge} 2\right]}\end{array}$ & $\begin{array}{c}\mathrm{q}_{0} \\
{\left[\mathrm{~W} / \mathrm{m}^{\wedge} 2\right]}\end{array}$ & $\begin{array}{c}\mathrm{T}_{2} \\
{\left[{ }^{\circ} \mathrm{C}\right]}\end{array}$ & $\begin{array}{c}\mathrm{q}_{2} \\
{\left[\mathrm{~W} / \mathrm{m}^{\wedge} 2\right]}\end{array}$ & & & \\
\hline Case1-w & $0.5 \mathrm{~S}_{0}$ & -86.44 & 59.95 & 179.8 & -112.84 & 25.84 & -47.62 & -53.57 & 5.95 \\
\hline Case 2-w & $0.8 \mathrm{~S}_{0}$ & -77.45 & 72.35 & 264.11 & -109.12 & 28.32 & -27.57 & -33.36 & 5.79 \\
\hline Case 3-w & $1.0 \mathrm{~S}_{0}$ & -73.2 & 78.85 & 318.55 & -107.25 & 29.64 & -18 & -22.67 & 4.67 \\
\hline
\end{tabular}

Table 1 Test conditions of SR107-ZK using solar simulator $(\mathrm{a}=0.17, \varepsilon=0.87)$ 
Table 2 Test conditions of Aluminum Kapton film using solar simulator $(\mathrm{a}=0.36, \quad \varepsilon=0.69)$

\begin{tabular}{|c|c|c|c|c|c|c|c|c|c|}
\hline & \multicolumn{4}{|c|}{ Faced side } & \multicolumn{2}{|c|}{ Reverse side } & \multirow[b]{2}{*}{$\begin{array}{c}\mathrm{T}_{\mathrm{s}}, \\
{\left[{ }^{\circ} \mathrm{C}\right]}\end{array}$} & \multirow[b]{2}{*}{$\begin{array}{c}\mathrm{T}_{\mathrm{s}} \\
{\left[{ }^{\circ} \mathrm{C}\right]}\end{array}$} & \multirow[b]{2}{*}{$\Delta \mathrm{T}$} \\
\hline & $\mathrm{S}$ & $\begin{array}{c}\mathrm{T}_{1} \\
{\left[{ }^{\circ} \mathrm{C}\right]}\end{array}$ & $\begin{array}{c}\mathrm{q}_{1} \\
{\left[\mathrm{~W} / \mathrm{m}^{\wedge} 2\right]}\end{array}$ & $\begin{array}{c}\mathrm{q}_{0} \\
{\left[\mathrm{~W} / \mathrm{m}^{\wedge} 2\right]}\end{array}$ & $\begin{array}{c}\mathrm{T}_{2} \\
{\left[{ }^{\circ} \mathrm{C}\right]}\end{array}$ & $\begin{array}{c}\mathrm{q}_{2} \\
{\left[\mathrm{~W} / \mathrm{m}^{\wedge} 2\right]}\end{array}$ & & & \\
\hline Case1-k & $0.5 \mathrm{~S}_{0}$ & -86.44 & 47.54 & 301.34 & -112.84 & 25.84 & -13.47 & -18.86 & 5.39 \\
\hline Case2-k & $0.8 \mathrm{~S}_{0}$ & -77.45 & 57.38 & 463.46 & -109.12 & 28.32 & 13.79 & 8.41 & 5.38 \\
\hline Case3-k & $1.0 \mathrm{~S}_{0}$ & -73.2 & 62.53 & 570.13 & -107.25 & 29.64 & 25.03 & 22.74 & 2.29 \\
\hline
\end{tabular}

Table 3 Test conditions of SR107-ZK using infrared cage ( $\mathrm{a}=0.17, \quad \varepsilon=0.87)$

\begin{tabular}{|c|c|c|c|c|c|c|c|c|c|}
\hline & \multicolumn{4}{|c|}{ Faced side } & \multicolumn{2}{|c|}{ Reverse side } & \multirow[b]{2}{*}{$\begin{array}{c}\mathrm{T}_{\mathrm{s}}{ }^{\prime} \\
{\left[{ }^{\circ} \mathrm{C}\right]}\end{array}$} & \multirow[b]{2}{*}{$\begin{array}{c}\mathrm{T}_{\mathrm{s}} \\
{\left[{ }^{\circ} \mathrm{C}\right]}\end{array}$} & \multirow[b]{2}{*}{$\Delta \mathrm{T}$} \\
\hline & $\mathrm{S}$ & $\begin{array}{c}\mathrm{q}_{0} \\
{\left[\mathrm{~W} / \mathrm{m}^{\wedge} 2\right]}\end{array}$ & Refer as & $\begin{array}{c}\mathrm{q}_{\mathrm{H}} \\
{\left[\mathrm{W} / \mathrm{m}^{\wedge} 2\right]}\end{array}$ & $\begin{array}{c}\mathrm{T}_{2} \\
{\left[{ }^{\circ} \mathrm{C}\right]}\end{array}$ & $\begin{array}{c}\mathrm{q}_{2} \\
{\left[\mathrm{~W} / \mathrm{m}^{\wedge} 2\right]}\end{array}$ & & & \\
\hline Case4 & $0.5 \mathrm{~S}_{0}$ & 179.8 & Case1-w & 206.66 & -97.74 & 37.03 & -46.58 & -50.64 & 4.06 \\
\hline Case5 & $0.8 \mathrm{~S}_{0}$ & 264.11 & Case1-w & 303.58 & -90.94 & 43.12 & -25.82 & -30.38 & 4.56 \\
\hline Case6 & $1.0 \mathrm{~S}_{0}$ & 318.55 & Case2-w & 366.15 & -86.73 & 47.25 & -14.91 & -19.56 & 4.65 \\
\hline
\end{tabular}

Table 4 Test conditions of Aluminum Kapton film using infrared cage $(a=0.36, \quad \varepsilon=0.69)$

\begin{tabular}{|c|c|c|c|c|c|c|c|c|c|}
\hline & \multicolumn{4}{|c|}{ Faced side } & \multicolumn{2}{|c|}{ Reverse side } & \multirow[b]{2}{*}{$\begin{array}{l}\mathrm{T}_{\mathrm{s}}{ }^{\prime} \\
{\left[{ }^{\circ} \mathrm{C}\right]}\end{array}$} & \multirow[b]{2}{*}{$\begin{array}{c}\mathrm{T}_{\mathrm{s}} \\
{\left[{ }^{\circ} \mathrm{C}\right]}\end{array}$} & \multirow[b]{2}{*}{$\Delta \mathrm{T}$} \\
\hline & $\mathrm{S}$ & $\begin{array}{c}\mathrm{q}_{0} \\
{\left[\mathrm{~W} / \mathrm{m}^{\wedge} 2\right]}\end{array}$ & Refer as & $\begin{array}{c}\mathrm{q}_{\mathrm{H}} \\
{\left[\mathrm{W} / \mathrm{m}^{\wedge} 2\right]}\end{array}$ & $\begin{array}{c}\mathrm{T}_{2} \\
{\left[{ }^{\circ} \mathrm{C}\right]}\end{array}$ & $\begin{array}{c}\mathrm{q}_{2} \\
{\left[\mathrm{~W} / \mathrm{m}^{\wedge} 2\right]}\end{array}$ & & & \\
\hline Case 7 & $0.5 \mathrm{~S}_{0}$ & 253.8 & Case 1-k & 436.73 & -84.2 & 49.87 & -8.98 & -14.31 & 5.33 \\
\hline Case8 & $0.8 \mathrm{~S}_{0}$ & 406.08 & Case2-k & 671.69 & -75.39 & 59.84 & 17.68 & 12.82 & 4.86 \\
\hline Case9 & $1.0 \mathrm{~S}_{0}$ & 507.6 & Case 3-k & 826.28 & -70.42 & 66.08 & 31.41 & 27.14 & 4.27 \\
\hline
\end{tabular}

\section{Conclusion}

Simulation of orbital heat flux plays a key role in the satellite thermal balance testing. Conclusions can be drawn from analysis: in thermal balance testing of micro satellite, in terms of simulating orbital heat flux, the results of two simulators are close enough to consider that it is feasible to choose infrared cage as orbital heat flux simulator, given coating material of typical $a / \varepsilon$ ratio. Results demonstrate that by using infrared cage simulator, the actual temperature is about $5{ }^{\circ} \mathrm{C}$ higher than theoretical value. The background heat flux interference stays stable at $17 \%$ of intensity of simulated orbital heat flux, and the value could be referred to in the model modification.

\section{References}

1. Gilimore D. Satellite Thermal Control. Second Edition. California: The Aerospace Corporation Press, 2002

2. Hou Zengqi and $\mathrm{Hu}$ Jingang, Spacecraft thermal control-theory and applications, Beijing: Chinese Technology Press, 2007, (in Chinese)

3. Baturkin Volodymyr. Micro-satellites thermal control--concepts and components. Acta Astronautica. 2005. 56(1-2): 161-170
4. Westheimer David T. Active Thermal Control System Considerations for the Next Generation of Human Rated Space Vehicles. AIAA-2005-342

5. Liu Jia. Analysis of Orbital Thermal Environment for Inclined-LEO Small Satellite, 2014 5th international conference on mechanical and aerospace engineering (ICMAE 2014). Madrid, Spain, 2014. 